\title{
The Determination of Type 2 Diabetes Mellitus's Impact on the Density of Retinal Blood Vessels and the Choriocapillaris: Optical Coherence Tomography Angiography Study
}

\author{
Marko Zlatanović, ${ }^{1}$ Jasmina Đorđević Jocić, ${ }^{1,2}$ Vesna Jakšić, ${ }^{3}$ Nevena Zlatanović, ${ }^{4}$ \\ Mladan Golubović, ${ }^{5}$ and Maja Živković (D) ${ }^{1,2}$ \\ ${ }^{1}$ Clinical Center Niš, Ophthalmology Clinic, Niš, Serbia \\ ${ }^{2}$ University of Niš, Faculty of Medicine, Department of Ophthalmology, Niš, Serbia \\ ${ }^{3}$ University of Belgrade, Medical Faculty, Department of Ophthalmology, Belgrade, Serbia \\ ${ }^{4}$ Community Health Center Niš in Niš, Niš, Serbia \\ ${ }^{5}$ University of Niš, Medical Faculty, Clinic for Anesthesiology and Intensive Care at the Clinical Center Niš in Nǐs, Nǐs, Serbia
}

Correspondence should be addressed to Maja Živković; drzivkovicmaja@gmail.com

Received 26 August 2021; Accepted 10 November 2021; Published 22 November 2021

Academic Editor: Marco Lupidi

Copyright (c) 2021 Marko Zlatanović et al. This is an open access article distributed under the Creative Commons Attribution License, which permits unrestricted use, distribution, and reproduction in any medium, provided the original work is properly cited.

\begin{abstract}
Optical coherence tomography angiography (OCTA) was used to analyze the alterations in the density of retinal blood vessels and the choriocapillaris (VD) in patients suffering from type 2 diabetes mellitus (T2DM). One hundred sixty-six eyes of 83 patients (43 of whom were men and 40 women, with a mean age of 58.59 \pm 14.04 ) with T2DM and without diabetic retinopathy were examined for the purpose of conducting the observational prospective study. The control group (CG) consisted of 66 eyes in 33 healthy subjects (15 male and 18 female, with a mean age of $55.12 \pm 12.70)$. The measurement regions of vessel density (VD) included the deep capillary plexus (DCP), the superficial capillary plexus (SCP), and the choriocapillaris. The results indicate considerable differences in the VD of the DCP and SCP when comparing the control group with the study groups $(p<0.001)$. In comparison with the control group $(p<0.001)$, there was a statistically significant reduction in the VD of the choriocapillaris in the study group. Furthermore, patients with T2DM showed a significantly decreased VD concerning the control in different macular regions. Thickness in several macular regions in the study group significantly decreased compared to the ones in the control group. OCTA was used to gather relevant information about the vascular changes which occurred in T2DM patients, assessed through the quantitative analysis of the blood flow in the retina and choriocapillaris.
\end{abstract}

\section{Introduction}

Type 2 diabetes mellitus, a metabolic disease of a complex and multifactorial nature, is characterized by the incapability of cells to level up glucose levels in the blood, as well as insulin resistance, leading to hyperglycemia, the main cause of diabetic complications [1-3]. Among many diabetic complications, one of particular interest is diabetic retinopathy (DR) [4-6]. Depending on several DR clinical features, which include hard exudates, cotton wool spots, macular edem, neovascularization hemorrhages, and microaneurysms, the classification of DR is twofold: (a) nonproliferative diabetic retinopathy (NPDR) and (b) proliferative diabetic retinopathy (PDR). NPDR, further divided into stages that can be mild, moderate, or severe, is a phase during which the retina does not show any new blood vessel growth. Unfortunately, DR is identified to be among the main causes of the loss of eyesight, and if not treated, DR will lead to visual impairment that cannot be regained; therefore, identifying microvascular changes before the clinical signs of DR become apparent at its early stage is preferable to action for preventing vision impairment $[7,8]$. 
With the aim of the quick creation of angiographic images, optical coherence tomography angiography (OCTA) uses information obtained from motion contrast imaging to high-resolution volumetric blood flow and is considered a noninvasive imaging technique. OCTA compares the decorrelation signals, defined as the differences in the amplitude or the intensity of the OCT signal that is backscattered, occurring between sequential OCT b-scans, and these must be performed at an identical cross section. In this manner, OCTA forms a blood flow map, strictly representing the movement of erythrocytes in the blood vessels of the retina [9-15].

The main aim of this study is to determine what kind of impact type 2 diabetes mellitus has on the density of the blood vessels of the retina and choriocapillaris with the application of optical coherence tomography angiography.

\section{Methods and Materials}

The present study involves the examination of two hundred and thirty-two eyes of 116 patients without DR, who reported to the Ophthalmologic Clinic, Clinical Center Niš, between March and July 2018. The inclusion criterion was age, ranging from 40 to 70 , with both genders equally included. The exclusion criteria were age-related macular degeneration, vascular diseases of the retina, glaucoma, myopia exceeding 6 diopters, history of panretinal laser photocoagulation after eye surgery, as well as other types of eye disorders, intravitreal administration of any drug, and significant lens opacity, for the purpose of avoiding reducedquality OCTA images. Sixty-six eyes belonging to 33 healthy subjects that were matched by age (15 men and 18 women, with a mean age of $55.12 \pm 12.70)$ whose ophthalmological examination yielded normal results and no eye diseases were used as the control group. The group of patients included 83 patients (166 eyes) suffering from type 2 diabetes mellitus without DR. Each patient went through a full eye exam, which included the following examinations: slit-lamp biomicroscopy, fundus examination, fundus autofluorescence (FAF), applanation tonometry, best-corrected visual acuity (BCVA) based on the Early Treatment of Diabetic Retinopathy Study (ETDRS), and OCTA (RTVue XR Avanti, AngioVue, Optovue, Inc., Freemont, CA). The participants in the study gave their informed written consent.

All the investigations respected the principles of the Declaration of Helsinki, and the Ethical Committee of the Faculty of Medicine, University of Niš, gave their approval for the mentioned.

\subsection{Optical Coherence Tomography Angiography. The} Optovue AngioVue System (ReVue XR software version 2017.1.0.151, issued by Optovue Inc., Fremont, CA, USA) was used to obtain OCTA images after conducting the standardized protocol whose basis is the SSADA (splitspectrum amplitude decorrelation algorithm) as detailed in the literature [12]. The classification of diabetic retinopathy according to the ETDRS was used as guidance for macular capillary network visualization, with scans performed centered on the fovea area $(3 \mathrm{~mm} \times 3 \mathrm{~mm})$ over the macular region divided into fovea, parafovea, and the full image. The automatic vascular network segmentation of the deep capillary plexus (DCP), superficial capillary plexus (SCP), and the choriocapillaris was performed with the application of the AngioAnalyticTM software. Vessel density (VD) was calculated by the same software and represented as the percentage of the microvasculature-occupied area and thickness in the total area of the scan and all of the sections. In this study, the definition of the choriocapillaris VD is the flow area divided by the selected area (radius was set to $1.5 \mathrm{~mm}$ ). Software that makes use of the $3 \mathrm{D}$ Projection Artifact Removal (PAR) algorithm was used for the purpose of improving OCTA image quality. We excluded from the analysis images with residual motion artifacts, low centration and focus, incorrect segmentation, and the index of the signal strength below 40 .

2.2. Statistical Analysis. The statistical analysis was done with SPSS 15 (SPSS Inc., Chicago, IL) for Windows. The Kruskall-Wallis test was used to analyze nonparametric data. The unpaired t-test was applied for the purpose of comparing the OCTA parameters between the control and test groups. It is considered that the $p$ value of $<0.05$ was of statistical significance.

\section{Results}

This study included 166 eyes of 83 patients (43 men $(51.8 \%)$ and 40 women $(48.2 \%)$ ) with a confirmed diagnosis of diabetes. The average age of the subjects in the diabetes group was $58.59 \pm 14.04$ (the mean age of the women was $59.10 \pm 15.20$, and that of the men was $58.10 \pm 12.92)$. Thus, men and women were practically the same age $(p=0.652)$. The control group (CG) consisted of 66 eyes in 33 subjects, of whom 15 (45.5\%) were men and 18 (54.5\%) were women. The average age of the subjects in this group was $55.12 \pm 12.70$ (the mean age of women was $55.28 \pm 12.71$, and that of men was $54.93 \pm 12.91)$. The men and women were of the same age $(p=0.914)$. The frequency of male respondents among patients with diabetes was $51.2 \%$, and in the CG, it was $45.5 \%$. $48.8 \%$ of the female respondents suffered from diabetes, and $54.5 \%$ had it in the CG. According to the proportion of male and female respondents, the groups (the group of patients and the CG) did not differ $(p=0.466)$.

According to the obtained results, retina superfical VD exhibits a much lower value $(p<0.001)$ in the group of patients $(41.9 \pm 6.8)$ than in the control group $(46.9 \pm 3.9)$. Retina deep VD is affected by whether or not the percipient has diabetes, since in the patient group, the mean value $(48.1 \pm 5.0)$ is statistically significantly lower $(p<0.001)$. Whether the patient has diabetes or not, does not significantly affect the change in retinal thickness for both the superfifical and the deep. The values obtained from the statistical analyses of the other obtained OCTA parameters are presented in Table 1.

The observation of the mean values of the VD of individual sectors of retina superficial showed that only the 
TABLE 1: Differences vessel density between the control and patient groups obtained with OCTA.

\begin{tabular}{|c|c|c|c|c|c|c|}
\hline & \multicolumn{4}{|c|}{ Density } & \multirow{2}{*}{\multicolumn{2}{|c|}{ Thickness }} \\
\hline & \multicolumn{2}{|c|}{ Retina superficial } & \multicolumn{2}{|c|}{ Retina deep } & & \\
\hline & Control group & Patient group & Control group & Patient group & Control group & Patient group \\
\hline Fovea & $17.5 \pm 7.7$ & $17.0 \pm 7.5$ & $31.3 \pm 7.1$ & $32.2 \pm 7.9$ & $324.0 \pm 29.4$ & $327.6 \pm 10.8$ \\
\hline Parafovea & $45.0 \pm 5.8$ & $50.1 \pm 4.2$ & $50.1 \pm 5.5$ & $55.8 \pm 2.9$ & $321.3 \pm 23.2$ & $327.6 \pm 10.8$ \\
\hline Superior hemi & $44.8 \pm 6.1$ & $49.5 \pm 5.4$ & $50.1 \pm 6.6$ & $55.9 \pm 3.0$ & $322.7 \pm 23.9$ & $328.3 \pm 12.5$ \\
\hline Inferior hemi & $45.2 \pm 5.8$ & $49.9 \pm 4.7$ & $49.8 \pm 5.6$ & $55.8 \pm 3.1$ & $319.4 \pm 23.1$ & $326.3 \pm 12.2$ \\
\hline Tempo & $43.6 \pm 6.3$ & $48.2 \pm 4.1$ & $49.9 \pm 5.6$ & $55.5 \pm 3.0$ & $315.5 \pm 22.4$ & $318.4 \pm 10.3$ \\
\hline Superior & $46.0 \pm 6.4$ & $50.7 \pm 5.4$ & $50.6 \pm 6.4$ & $56.1 \pm 3.3$ & $324.9 \pm 24.5$ & $332.1 \pm 11.7$ \\
\hline Nasal & $44.7 \pm 6.0$ & $49.6 \pm 4.8$ & $50.4 \pm 6.5$ & $56.0 \pm 2.7$ & $325.0 \pm 26.2$ & $329.5 \pm 14.9$ \\
\hline Inferior & $46.2 \pm 6.2$ & $51.5 \pm 4.8$ & $49.3 \pm 6.5$ & $55.5 \pm 3.7$ & $319.8 \pm 24.9$ & $327.3 \pm 14.6$ \\
\hline
\end{tabular}

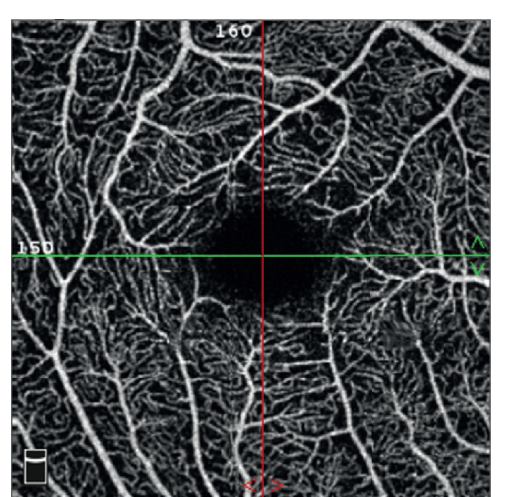

Superficial (ILM - IPL)

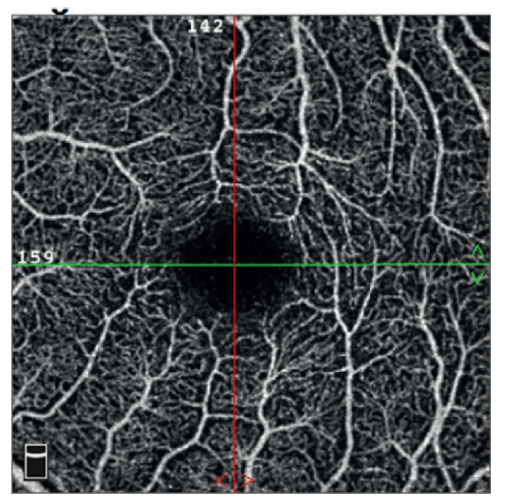

Superficial (ILM - IPL)

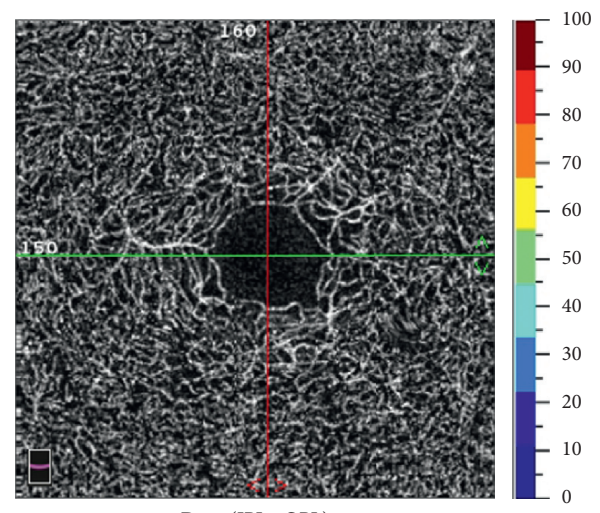

Deep (IPL - OPL)

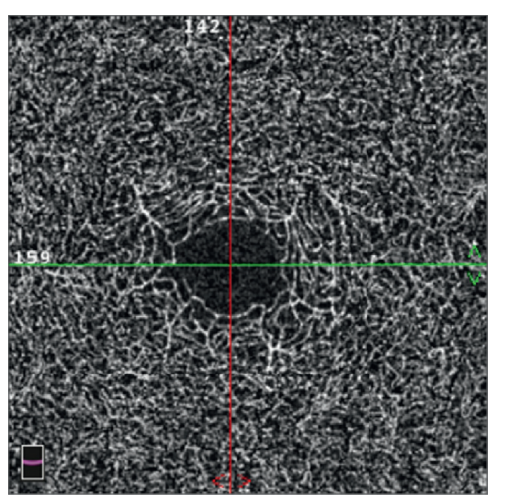

Deep (IPL - OPL)

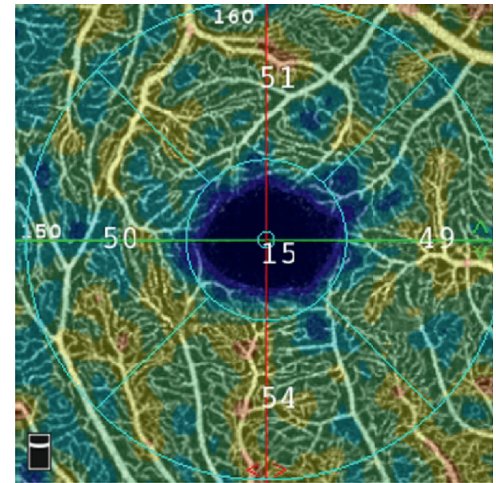

Vessel Density (Superficial)

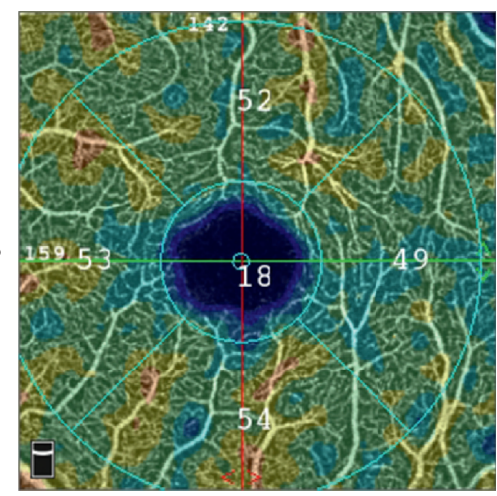

Vessel Density (Superficial)

FIGURE 1: Optical coherence tomography angiography (OCTA) of the retina from a patient suffering from type 2 diabetes (a) and a subject who is healthy (b).

value of the fovea was the same both in the group with patients and in the control group. All other sectors-parafovea $(45.0 \pm 5.8)$, superior hemi $(44.8 \pm 6.1)$, inferior hemi (45.2 \pm 5.8$)$, tempo $(43.6 \pm 6.3)$, superior $(46.0 \pm 6.4)$, nasal $(44.7 \pm 6.0)$, and inferior $(46.2 \pm 6.2)-$ show a significantly reduced (obtained $p$ value was $<0.001$ ) values compared to the control group, where the average values were the following: parafovea $(50.1 \pm 4.2)$, superior hemi $(49.5 \pm 5.4)$, inferior hemi $(49.9 \pm 4.7)$, tempo $(48.2 \pm 4.1)$, superior $(50.7 \pm 5.4)$, nasal $(49.6 \pm 4.8)$, and inferior $(51.5 \pm 4.8)$.

There is no considerable difference of the average values of the retina deep fovea sector when we compare the group with patients and the control group, as is the case with the same retina superficial sector. All the other sectors-parafovea (50.1 \pm 5.5$)$, superior hemi $(50.1 \pm 6.6)$, inferior hemi (49.8 \pm 5.6$)$, tempo ( $49.9 \pm 5.6)$, superior ( $50.6 \pm 6.4$ ), nasal (50.4 \pm 6.5$)$, and inferior $(49.3 \pm 6.5)$ - have a much more reduced (obtained $p$ value was $<0.001$ ) value than those of the control group does, which exhibits the following values: parafovea $(55.8 \pm 2.9)$, superior hemil $(55.9 \pm 3.0)$, inferior hemi (55.8 \pm 3.1$)$, tempo ( $55.5 \pm 3.0)$, superior ( $56.1 \pm 3.3$ ), nasal $(56.0 \pm 2.7)$, and inferior $(55.5 \pm 3.7)$. The images obtained from OCTA regarding the VD of a patient with T2DM and a healthy individual are shown in Figure 1.

There were no statistically significant differences in all the average values of the retinal thickness sectors between the CG and the patient group. The images obtained from 

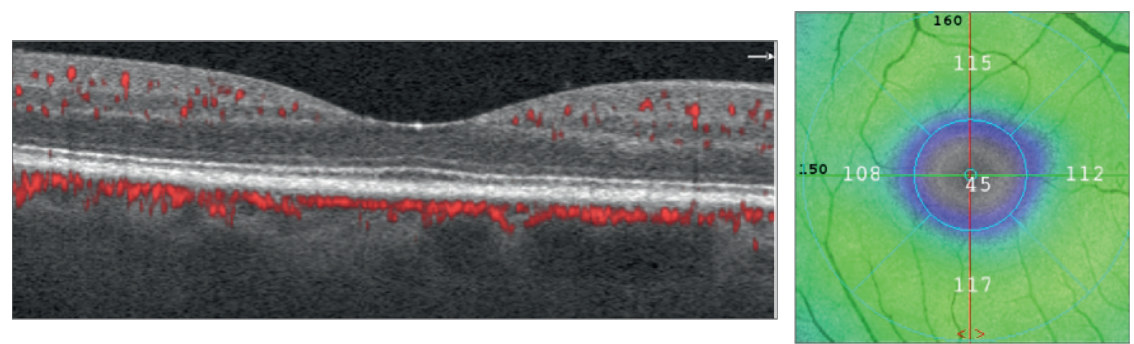

Inner Thickness (ILM - IPL)
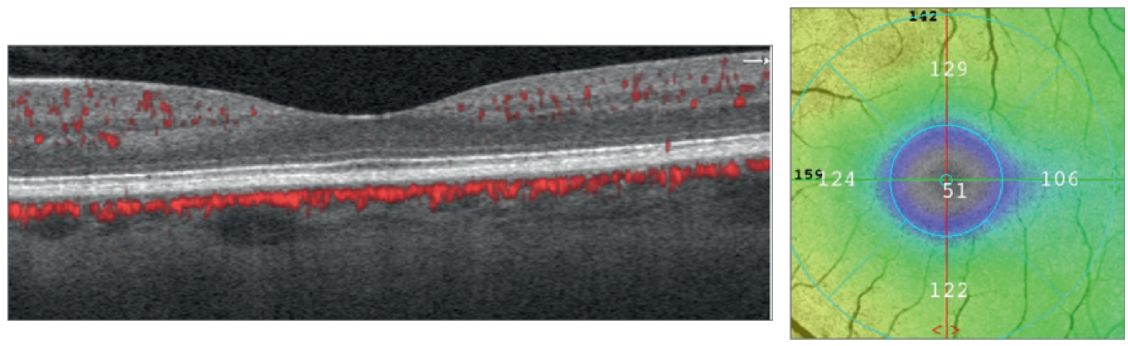

Inner Thickness (ILM - IPL)

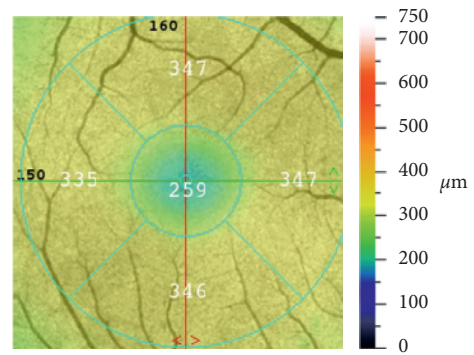

Full Thickness (ILM - RPE)

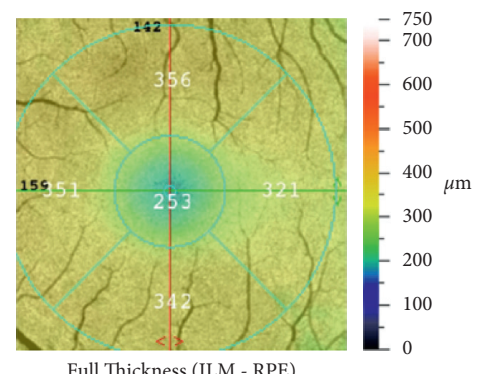

Full Thickness (ILM - RPE)

FIGURE 2: The thickness of the retina obtained by OCTA from a patient suffering from type 2 diabetes (abovementioned) and a subject who is healthy (below).
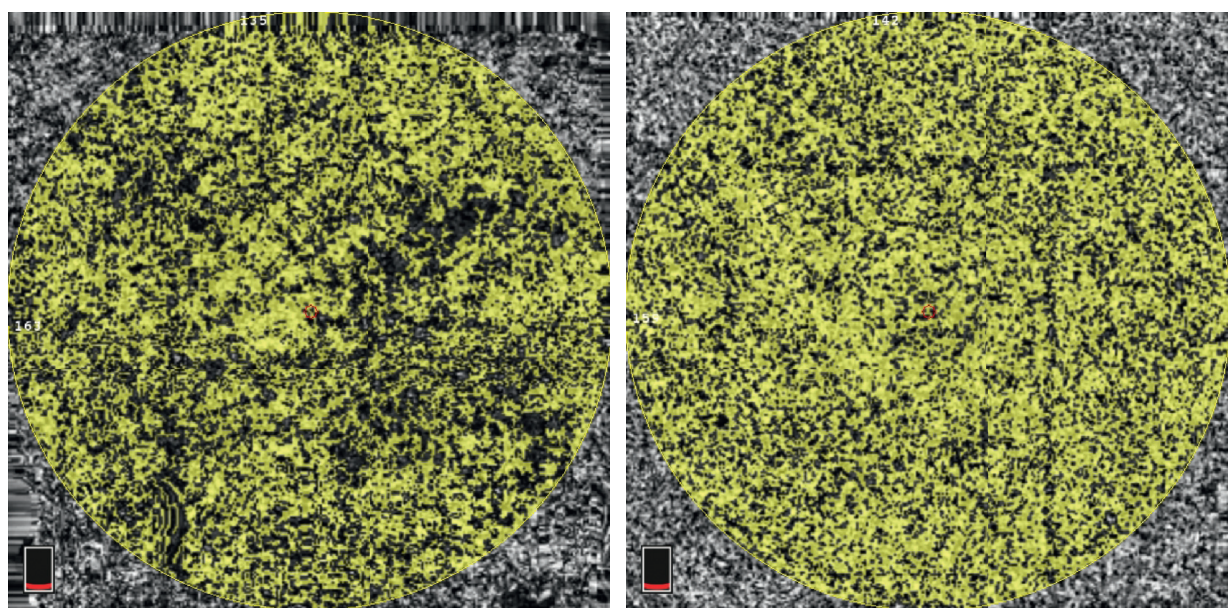

Figure 3: Choriocapillaris obtained by OCTA of the retina from a patient suffering from type 2 diabetes (a) and a subject who is healthy (b).

OCTA regarding the thickness from a patient with T2DM and healthy individuals are shown in Figure 2.

According to the obtained results regarding choriocapillaris, the flow area was much lower $(p<0.001)$ in the patient group (4.622 \pm 0.389$)$ than that in the control group $(4.791 \pm 0.252)$. The same observation was valid for choriocapillaris $\mathrm{VD}$, where the stated parameter was much lower $(p<0.001)$ in the patient group $(0.642 \pm 0.097)$ than that in the control group $(0.678 \pm 0.035)$. The images obtained from OCTA regarding choriocapillaris from a patient with T2DM and healthy individuals are shown in Figure 3.

\section{Discussion}

Although DR is treated as a complex condition, recent studies show that DR could be related to microvascular abnormality development [16, 17]. For this reason, microvascular abnormality development in the vascular layers of the retina was evaluated in this study with the application of OCTA, and it was indicated with superficial and deep VD. Previous studies suggested that a combination of reduced VD, accompanied by increased FAZ area, could be considered as parameters used to detect DR at an early stage $[18,19]$. Lupidi et al. published a comparative analysis that compared groups of diabetics and healthy people, demonstrating differences that are of a statistical significance considering the major axis, surface, and perimeter of the FAZ, owing to the FAZ enlargement found in diabetic maculopathy, both of superficial and deep capillary plexuses, suggesting that progressive nonperfusion was not limited to a particular layer in diabetic patients [20].

The findings reported by Dimitrova et al. show that the density of superficial capillaries and deep capillaries has 
decreased in the parafoveal area of the retina and the choriocapillaris in T2DM patients that do not have DR [19]. In addition, Simonett et al. reported that there is a notable decrease in the density of the parafoveal vessel within the deep capillary plexus, detected in patients suffering from T1DM and those that have mild DR [21]. Our study supports these findings since we have discovered that there is a significant difference in the VD parameters of the retina superficial and the retina deep, both exhibiting lower values in the patient group. This process could be associated with the larger extent of microaneurysms in the deep vascular plexus than those in the superficial plexus [22-25]. Previous studies related to retinal thickness, based on OCT, presented controversial results, such as the one where no significant difference was found in the thickness concerning both the group of patients and healthy controls, and another where there was a notable reduction in thickness present in patients suffering from diabetes without DR or those with mild NPDR when compared to the healthy population $[26,27]$. Our study does not reveal any significant difference difference in the OCTA parameters related to retina thickness between the groups.

According to Lupidi et al. subjects suffering from diabetes were observed to have a significant negative linear correlation between CC VPD and DCP VPD. However, the correlation between CC VPD and SCP VPD was insignificant. The authors reported further that there was a positive linear correlation between SCP, or CC VPD and DCP VPD, in healthy subjects. According to the obtained results, Lupidi et al. suggested that there could be a functional interconnectedness between the choroidal vascular networks and those of the retina based on the hypothesis that, in terms of VPD, the negative linear correlation between DCP and CC could express a potential mechanism for compensation [28]. The DCP-level blood flow may slightly increase for the purpose of supplying the outer layers of the retina in the event of CC insufficiency, while the CC perfusion may increase with the aim of compensating for the demand for oxygen and the metabolic demand in case retinal capillary impairment occurs, which often includes the DCP in the case of DR $[18,29]$.

Based on the current information at our disposal, we believe that no other study has observed changes in the different parts of both the superficial and the deep retina (parafovea, fovea, inferior hemi, superior hemi, tempo, inferior, superior, and nasal). According to the obtained results for both superficial and deep retina, only the value of the fovea is the same both in the group of patients and in the control group. The value for retina thickness did not differ between the patient and healthy population groups. The complex nature of diabetes and both vascular and neurodegenerative changes prevents us from giving a possible explanation for this.

Different studies related to choriocapillaris reported controversial findings. Impairments of the focal and diffuse CC flow in the eyes of patients suffering from diabetes were reported by Choi et al. while Nesper et al. [30, 31] reported an increased CC nonperfusion area in the eyes of the patients suffering from diabetes without retinopathy, compared to the ones of the control group. Other studies $[19,32]$ have reported no notable differences in the density of the CC vessel when comparing eyes of diabetics without retinopathy and those of healthy controls. According to the findings presented in this research, both the choriocapillaris flow area and the choriocapillaris VD were much lower in the group of patients than in the control group, and these results are supported by previous histopathological studies [33].

The presented study has several limitations related to a single-center analysis (all subjects were of the same ethnicity) and single time-point (data were taken once; therefore, the evaluation of changes over time is not possible).

In summary, the results presented in this study indicate that OCT angiography parameters associated with the density of the superficial and deep vessels of the retina have lower values in patients suffering from diabetes without DR than in healthy subjects. One of the reasons for these differences could be the autoregulation alternation of the retinal blood vessels in patients suffering from diabetes without DR, further associated with a patient's systemic characteristics. According to the obtained results, OCT angiography could be considered as a potential biomarker used for the risk of DR development in patients suffering from diabetes without DR evaluation.

\section{Data Availability}

The authors confirm that the data supporting the findings of this study are available within the article.

\section{Conflicts of Interest}

The authors declare that they have no conflicts of interest.

\section{References}

[1] C. N. Hales and D. J. P. Barker, "Type 2 (non-insulin-dependent) diabetes mellitus: the thrifty phenotype hypothesis," Diabetologia, vol. 35, no. 7, pp. 595-601, 1992.

[2] M. Y. Donath and S. E. Shoelson, "Type 2 diabetes as an inflammatory disease," Nature Reviews Immunology, vol. 11, no. 2, pp. 98-107, 2011.

[3] A. R. Saltiel and C. R. Kahn, "Insulin signalling and the regulation of glucose and lipid metabolism," Nature, vol. 414, no. 6865, pp. 799-806, 2001.

[4] K. Dahl-Jørgensen, O. Brinchmann-Hansen, H.-J. Bangstad, and K. F. Hanssen, "Blood glucose control and microvascular complications - what do we do now?” Diabetologia, vol. 37, no. 12, pp. 1172-1177, 1994.

[5] K. Papatheodorou, M. Banach, M. Edmonds, N Papanas, and D Papazoglou, "Complications of diabetes," Journal of diabetes research, vol. 2015, Article ID 189525, 2015.

[6] T. M. Diep and I. Tsui, "Risk factors associated with diabetic macular edema," Diabetes Research and Clinical Practice, vol. 100, no. 3, pp. 298-305, 2013.

[7] A. Patz, "Studies on retinal neovascularization," Friedenwald Lecture Investigative Ophthalmology \& Visual Science, vol. 19, no. 10, pp. 1133-1138, 1980.

[8] L. Giancardo, F. Meriaudeau, T. P. Karnowski et al., "Exudatebased diabetic macular edema detection in fundus images 
using publicly available datasets," Medical Image Analysis, vol. 16, no. 1, pp. 216-226, 2012.

[9] T. E. de Carlo, A. Romano, N. K. Waheed, and J. S. Duker, "A review of optical coherence tomography angiography (OCTA)," International Journal of Retina and Vitreous, vol. 1, no. 1, p. 5, 2015.

[10] Z. Sun, D. Yang, Z. Tang, D. S. Ng, and C. Y. Cheung, "Optical coherence tomography angiography in diabetic retinopathy: an updated review," Eye, vol. 35, no. 1, pp. 149-161, 2021.

[11] H. Khalid, R. Schwartz, L. Nicholson et al., "Widefield optical coherence tomography angiography for early detection and objective evaluation of proliferative diabetic retinopathy," British Journal of Ophthalmology, vol. 105, no. 1, pp. 118-123, 2021.

[12] M. Lupidi, F. C. Piccolino, R. Corbucci, and C. Cagini, "Optical coherence tomography angiography," Jay Chhablani, Central Serous Chorioretinopathy, Academic Press, Cambridge, MA, USA, pp. 129-143, 2019.

[13] G. Coscas, M. Lupidi, F. Coscas, J. Chhablani, and C. Cagini, "Optical coherence tomography angiography in healthy subjects and diabetic patients," Ophthalmologica, vol. 239, no. 2-3, pp. 61-73, 2018.

[14] Y. Jia, O. Tan, J. Tokayer et al., "Split-spectrum amplitudedecorrelation angiography with optical coherence tomography," Optics Express, vol. 20, no. 4, pp. 4710-4725, 2012.

[15] M. D. Abramoff, P. E. Fort, I. C. Han, K. T. Jayasundera, E. H. Sohn, and T. W. Gardner, "Approach for a clinically useful comprehensive classification of vascular and neural aspects of diabetic retinal disease," Investigative Opthalmology \& Visual Science, vol. 59, no. 1, pp. 519-527, 2018.

[16] A. Verma, R. Raman, K. Vaitheeswaran et al., "Does neuronal damage precede vascular damage in subjects with type 2 diabetes mellitus and having no clinical diabetic retinopathy?" Ophthalmic Research, vol. 47, no. 4, pp. 202-207, 2012.

[17] M. Ashraf, P. L. Nesper, L. M. Jampol, F. Yu, and A. A. Fawzi, "Statistical model of optical coherence tomography angiography parameters that correlate with severity of diabetic retinopathy," Investigative Opthalmology \& Visual Science, vol. 59, no. 10, pp. 4292-4298, 2018.

[18] A. Carnevali, R. Sacconi, E. Corbelli et al., "Optical coherence tomography angiography analysis of retinal vascular plexuses and choriocapillaris in patients with type 1 diabetes without diabetic retinopathy," Acta Diabetologica, vol. 54, no. 7, pp. 695-702, 2017.

[19] G. Dimitrova, E. Chihara, H. Takahashi, H. Amano, and K. Okazaki, "Quantitative retinal optical coherence tomography angiography in patients with diabetes without diabetic retinopathy," Investigative Opthalmology \& Visual Science, vol. 58, no. 1, pp. 190-196, 2017.

[20] M. Lupidi, G. Coscas, F. Coscas et al., "Retinal microvasculature in nonproliferative diabetic retinopathy: automated quantitative optical coherence tomography angiography assessment," Ophthalmic Research, vol. 58, no. 3, pp. 131-141, 2017.

[21] J. M. Simonett, F. Scarinci, F. Picconi et al., "Early microvascular retinal changes in optical coherence tomography angiography in patients with type 1 diabetes mellitus," Acta Ophthalmologica, vol. 95, no. 8, pp. e751-e755, 2017.

[22] A. Couturier, V. Mané, S. Bonnin et al., "Capillary plexus anomalies in diabetic retinopathy on optical coherence tomography angiography," Retina, vol. 35, no. 11, pp. 2384-2391, 2015.

[23] N. Hasegawa, M. Nozaki, N. Takase, M. Yoshida, and Y. Ogura, "New insights into microaneurysms in the deep capillary plexus detected by optical coherence tomography angiography in diabetic macular edema," Investigative Opthalmology \& Visual Science, vol. 57, no. 9, pp. OCT348OCT355, 2016.

[24] F. Scarinci, L. M. Jampol, R. A. Linsenmeier, and A. A. Fawzi, "Association of diabetic macular nonperfusion with outer retinal disruption on optical coherence tomography," JAMA Ophthalmology, vol. 133, no. 9, pp. 1036-1044, 2015.

[25] A. Ishibazawa, T. Nagaoka, A. Takahashi et al., "Optical coherence tomography angiography in diabetic retinopathy: a prospective pilot study," American Journal of Ophthalmology, vol. 160, no. 1, pp. 35-44, 2015.

[26] P. S. Saif, A. E.-R. G. Salman, N. A. H. Omran, and Y. A. T. Farweez, "Assessment of diabetic retinopathy vascular density maps,” Clinical Ophthalmology, vol. 14, pp. 3941-3953, 2020.

[27] C. Shen, S. Yan, M. Du, H. Zhao, L. Shao, and Y. Hu, “Assessment of capillary dropout in the superficial retinal capillary plexus by optical coherence tomography angiography in the early stage of diabetic retinopathy," BMC Ophthalmology, vol. 18, no. 1, p. 113, 2018.

[28] M. Lupidi, A. Cerquaglia, R. Gujar et al., "Functional correlation between choroidal and retinal vascularity in lowgrade diabetic retinopathy," Acta Diabetologica, vol. 57, no. 8, pp. 983-990, 2020.

[29] F. Scarinci, P. L. Nesper, and A. A. Fawzi, "Deep retinal capillary nonperfusion is associated with photoreceptor disruption in diabetic macular ischemia," American Journal of Ophthalmology, vol. 168, pp. 129-138, 2016.

[30] W. Choi, K. J. Mohler, B. Potsaid et al., "Choriocapillaris and choroidal microvasculature imaging with ultrahigh speed OCT angiography," PLoS One, vol. 8, no. 12, Article ID e81499, 2013.

[31] P. L. Nesper, B. T. Soetikno, and A. A. Fawzi, "Choriocapillaris nonperfusion is associated with poor visual acuity in eyes with reticular pseudodrusen," American Journal of Ophthalmology, vol. 174, pp. 42-55, 2017.

[32] F. F. Conti, V. L. Qin, E. B. Rodrigues et al., "Choriocapillaris and retinal vascular plexus density of diabetic eyes using splitspectrum amplitude decorrelation spectral-domain optical coherence tomography angiography," British Journal of Ophthalmology, vol. 103, no. 4, pp. 452-456, 2019.

[33] D. S. McLeod and G. A. Lutty, "High-resolution histologic analysis of the human choroidal vasculature," Investigative Ophthalmology \& Visual Science, vol. 35, pp. 3799-3811, 1994. 